\title{
Are all voices heard in the COVID-19 debate?
}

\author{
Stan Benjamens ${ }^{1}$ (D) . Vincent E. de Meijer ${ }^{1}$ (D) $\cdot$ Robert A. Pol $^{1}$ (D) Martijn P. D. Haring ${ }^{1}$ (D)
}

Received: 30 August 2020 / Accepted: 22 September 2020 / Published online: 10 October 2020

(c) Akadémiai Kiadó, Budapest, Hungary 2020

\begin{abstract}
The COVID-19 pandemic has vast global consequences. Yet, effective mitigation strategies and economic and medical outfall differ extensively across the globe. It is currently unclear how well researchers from all continents are represented in the unsolicited and solicited publications. A literature review was performed in SCOPUS on COVID-19 oriented publications in the four most impactful medical journals. These included the British Medical Journal, Journal of the American Medical Association, the New England Journal of Medicine and The Lancet. We identified 809 eligible publications out of identified 924 records. The vast majority of publications on COVID-19, in the four can be considered European $(47.7 \%)$ or North-American $(37.3 \%)$ research. Chinese reports were relatively common (8.8\%); however, reports from other Asian countries (3.2\%) were minimal. Research from the African (1.0\%) and South-American continents (0.6\%) was rarely published in these journals. These observations are not surprising, as they reflect global academic publishing. However, involving all continents into COVID-19 research is important as COVID-19 management strategies and societal and economic consequences differ extensively across the globe. We see an important role for medical journals in encouraging global voices through solicited articles, to ensure a weighted research and humanitarian response.
\end{abstract}

Keywords Global health · COVID-19 · Literature review

\section{Introduction}

The global fight against the current COVID-19 pandemic warrants fast and reliable distribution of new insights into the direct and indirect health consequences. The prompt need of robust and reliable scientific data resulted in an overwhelming number of scientific publications. Though, a measured and comprehensive global response requires a balanced distribution of publications.

Electronic supplementary material The online version of this article (https://doi.org/10.1007/s1119 2-020-03730-z) contains supplementary material, which is available to authorized users.

Stan Benjamens

s.benjamens@umcg.nl

1 Department of Surgery, University of Groningen, University Medical Center Groningen, Groningen, The Netherlands 


\section{Methods}

The Scopus database was systematically searched for COVID-19 oriented publications published from December 31, 2019, to July 27, 2020, following the Preferred Reporting Items for Systematic Reviews and Meta-analysis (PRISMA) statements checklist and flowchart (Supplement). The query included "COVID-19" (all text) and was limited the four most impactful medical journals: The New England Journal of Medicine; The Lancet; the Journal of the American Medical Association; and the British Medical Journal. All articles authored by a member of the editorial board or by the journal's writing team were excluded. Publications were stratified into two groups, being unsolicited publications and solicited publications, based on information provided in the Author Guidelines of the specific journal. The country and continent of origin was based on the corresponding authors' affiliation. To compose Fig. 1, weighted-lines (based on the total number of publications per continent) were drawn between the continents and journals.

\section{Results}

A total of 809 COVID-19 oriented publications were identified: 125 in the New England Journal of Medicine; 203 in The Lancet; 166 in the Journal of the American Medical Association; and 315 in the British Medical Journal. The vast majority of publications can be considered European (47.7\%) or North-American (37.3\%) research (Fig. 1). Especially researchers from the United States of America and the United Kingdom are well represented, covering $35.6 \%$ and $35.0 \%$ of publications respectively. Chinese reports were relatively common (8.8\%); however, reports from other Asian countries (3.2\%) were minimal.

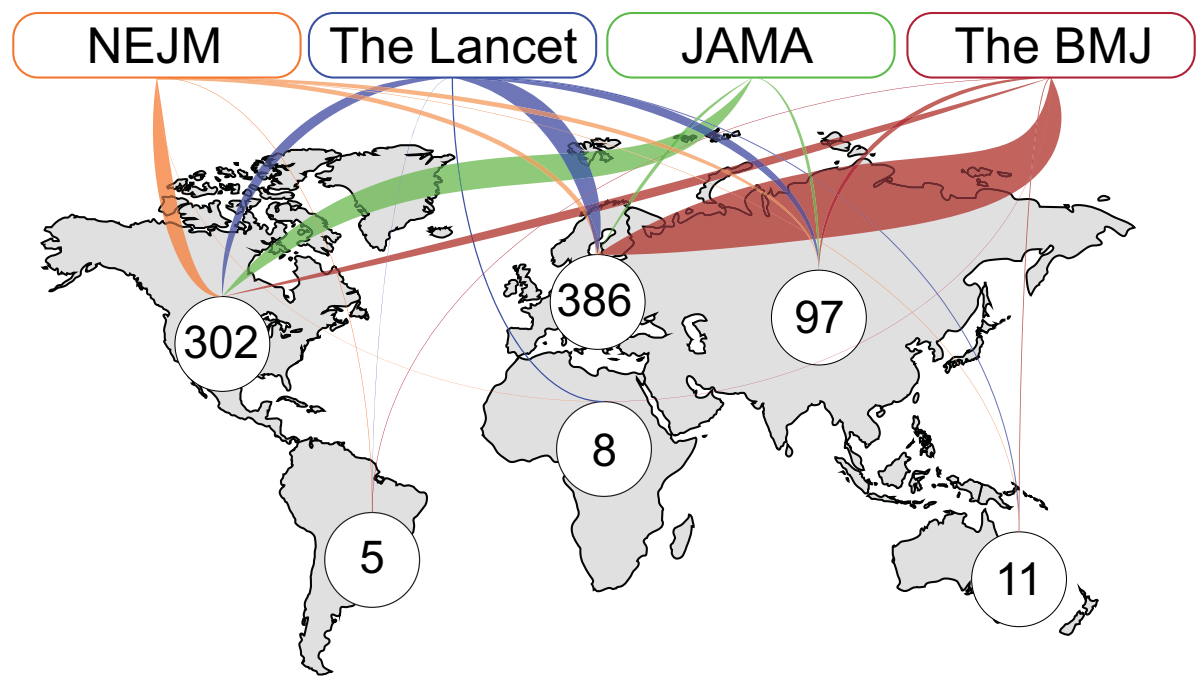

Fig. 1 World map of COVID-19 oriented publications. Publication output per for the four most impactful medical journals. Line weight calculated on number of publications (Supplementary Material). Abbreviations: The New England Journal of Medicine, NEJM; the Journal of the American Medical Association, JAMA; the British Medical Journal, The BMJ 
Research from the African (1.0\%) and South-American continents $(0.6 \%)$ was rarely published in these journals. A similar distribution was found among solicited articles, with a clear European (36.6\%) and North-American (51.7\%) predominance of likely solicited publications.

\section{Discussion}

The current observations are not surprising, as they reflect global academic publishing (Conte et al. 2017). This analysis does not regard subject matter, and research on consequences of COVID-19 in for example Africa and South-America may be initiated by authors from Europe and North-America. However, involving all continents into COVID19 research is extremely important. For instance the African continent and small island nations are at increased risk due to a high HIV/AIDS and diabetes mellitus prevalence (Clark et al. 2020). And the number of new cases and fatalities is on the rise in SouthAmerica (World Health Organization 2020). Both continents, mostly comprising lowand middle-income countries, face a higher impact of indirect consequences compared to Europe and North-America. Estimations are that up to one-million child deaths and up to fifty-thousand additional maternal deaths could result from a lack of essential health coverage (Roberton et al. 2020). The differences in demographics, social contact patterns, health care availability and quality, and comorbidity distribution, result in alternating mortality differences between age groups and lower effectiveness of mitigation strategies (Hopman et al. 2020; Walker et al. 2020). The importance of these region specific strategies is affirmed by research on the effect of population heterogeneity on herd immunity, which can possibly already be achieved with a population-wide infection rate of $\sim 40 \%$ because of age associated contact patterns (Britton et al. 2020).

Although performance of research may be skewed due to the global distribution of research facilities, reflecting upon these findings asks for global representation since it concerns a global problem. The international response to the current pandemic as well as to the potential second wave should be a joint effort, and hearing voices from continents facing unprecedented consequences, such as Africa and South-America, should guide further actions. We see an important role for medical journals in encouraging global voices, to ensure a weighted research and humanitarian response.

Author contributions All authors contributed to the study conception and design. Material preparation, data collection and analysis were performed by Stan Benjamens and Martijn haring. The first draft of the manuscript was written by Stan Benjamens and Martijn Haring and all authors commented on previous versions of the manuscript. The figures were designed by Stan Benjamens and Martijn Haring. All authors read and approved the final manuscript.

Funding The authors have no funding to be reported for this manuscript

Availability of data and material Search query and results of literature study were incuded in the supplementary materials.

\section{Compliance with ethicla standards}

Conflict of interest The authors declare that they have no conflict of interest.

Ethics approval Not applicable. 
Consent to participate Not applicable.

Consent for publication: Not applicable.

Code availability Not applicable.

\section{References}

Britton, T., Ball, F., \& Trapman, P. (2020). A mathematical model reveals the influence of population heterogeneity on herd immunity to SARS-CoV-2. Science, 369(6505), 846-849. https://doi.org/10.1126/ science.abc6810.

Clark, A., Jit, M., Warren-Gash, C., Guthrie, B., Wang, H. H. X., Mercer, S. W., et al. (2020). Global, regional, and national estimates of the population at increased risk of severe COVID-19 due to underlying health conditions in 2020: A modelling study. The Lancet Global Health, 8(8), e1003-e1017. https://doi.org/10.1016/S2214-109X(20)30264-3.

Conte, M. L., Liu, J., Schnell, S., \& Omary, M. B. (2017). Globalization and changing trends of biomedical research output. JCI Insight. https://doi.org/10.1172/jci.insight.95206.

Hopman, J., Allegranzi, B., \& Mehtar, S. (2020). Managing COVID-19 in low- and middle-income countries. JAMA, 323(16), 1549. https://doi.org/10.1001/jama.2020.4169.

Roberton, T., Carter, E. D., Chou, V. B., Stegmuller, A. R., Jackson, B. D., Tam, Y., et al. (2020). Early estimates of the indirect effects of the COVID-19 pandemic on maternal and child mortality in lowincome and middle-income countries: A modelling study. The Lancet Global Health, 8(7), 901-909. https://doi.org/10.1016/S2214-109X(20)30229-1.

Walker, P. G. T., Whittaker, C., Watson, O. J., Baguelin, M., Winskill, P., Hamlet, A., et al. (2020). The impact of COVID-19 and strategies for mitigation and suppression in low- and middle-income countries. Science. https://doi.org/10.1126/science.abc0035.

World Health Organization. (2020). Coronavirus disease (COVID-19): Situation Report-191.

Publisher's Note Springer Nature remains neutral with regard to jurisdictional claims in published maps and institutional affiliations. 